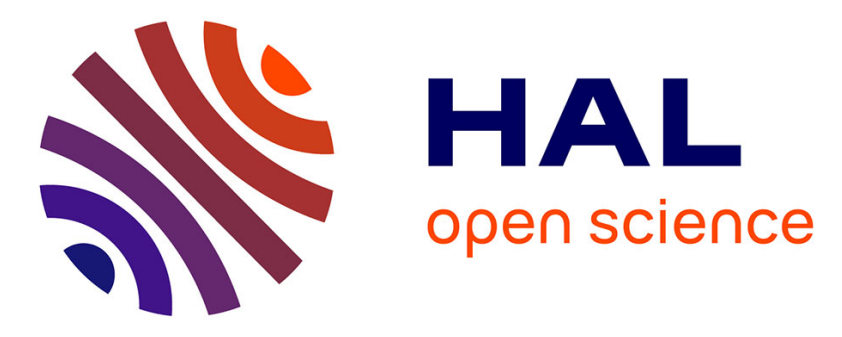

\title{
Sparse classification with MRI based markers for neuromuscular disease categorization
}

Katerina Gkirtzou, Jean-François Deux, Guillaume Bassez, Aristeidis Sotiras, Alain Rahmouni, Thibault Varacca, Nikos Paragios, Matthew Blaschko

\section{- To cite this version:}

Katerina Gkirtzou, Jean-François Deux, Guillaume Bassez, Aristeidis Sotiras, Alain Rahmouni, et al.. Sparse classification with MRI based markers for neuromuscular disease categorization. 4th International Workhop on Machine Learning in Medical Imaging, Sep 2013, Nagoya, Japan. hal00845126

\section{HAL Id: hal-00845126 \\ https://hal.inria.fr/hal-00845126}

Submitted on 18 Jul 2013

HAL is a multi-disciplinary open access archive for the deposit and dissemination of scientific research documents, whether they are published or not. The documents may come from teaching and research institutions in France or abroad, or from public or private research centers.
L'archive ouverte pluridisciplinaire HAL, est destinée au dépôt et à la diffusion de documents scientifiques de niveau recherche, publiés ou non, émanant des établissements d'enseignement et de recherche français ou étrangers, des laboratoires publics ou privés. 


\title{
Sparse classification with MRI based markers for neuromuscular disease categorization
}

\author{
Katerina Gkirtzou ${ }^{1,2}$, Jean-François Deux ${ }^{3}$, Guillaume Bassez ${ }^{3}$, Aristeidis \\ Sotiras $^{4}$, Alain Rahmouni ${ }^{3}$, Thibault Varacca ${ }^{3}$, Nikos Paragios ${ }^{1,2}$, and \\ Matthew B. Blaschko ${ }^{1,2}$ * \\ 1 Center for Visual Computing, École Centrale Paris, France \\ 2 Équipe Galen, INRIA Saclay, Île-de-France, France \\ 3 Henri Mondor University Hospital, Créteil, France \\ 4 Department of Radiology, University of Pennsylvania, USA
}

\begin{abstract}
In this paper, we present a novel method for disease classification between two patient populations based on features extracted from Magnetic Resonance Imaging (MRI) data. Anatomically meaningful features are extracted from structural data (T1- and T2-weighted MR images) and Diffusion Tensor Imaging (DTI) data, and used to train a new machine learning algorithm, the $k$-support SVM ( $k$ sup-SVM). The $k$ support regularized SVM has an inherent feature selection property, and thus it eliminates the requirement for a separate feature selection step. Our dataset consists of patients that suffer from facioscapulohumeral muscular dystrophy (FSH) and Myotonic muscular dystrophy type 1 (DM1) and our proposed method achieves a high performance. More specifically, it achieves a mean Area Under the Curve (AUC) of 0.7141 and mean accuracy $77 \% \pm 0.013$. Moreover, we provide a sparsity visualization of the features in order to indentify their discriminative value. The results suggest the potential of the combined use of MR markers to diagnose myopathies, and the general utility of the $k$ sup-SVM. Source code is also available at https://gitorious.org/ksup-svm.
\end{abstract}

Keywords: myopathies, Diffusion Tensor Imaging, $k$-support regularized SVM

\section{Introduction}

In this paper, we tackle the problem of disease classification. More specifically, we are interested in discriminating between two different neuromuscular diseases, facioscapulohumeral muscular dystrophy (FSH) and myotonic muscular dystrophy type 1 (DM1). Myopathies result in an atrophy and weakness of the muscle, and currently require an invasive biopsy to distinguish the two. In contrast, we pursue here a comparatively non-invasive approach based on MR imaging. Our approach to discriminate between the two diseases is centered around two axes:

* This work was partially funded by ERC Grant 259112 and the AFM-Telethon foundation. 


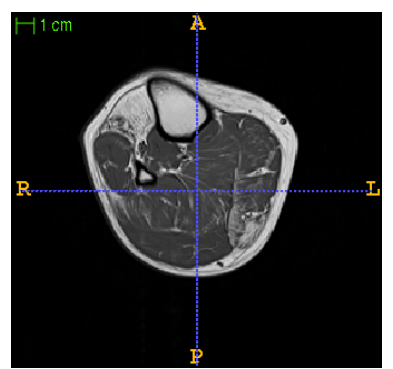

(a) FSH patient

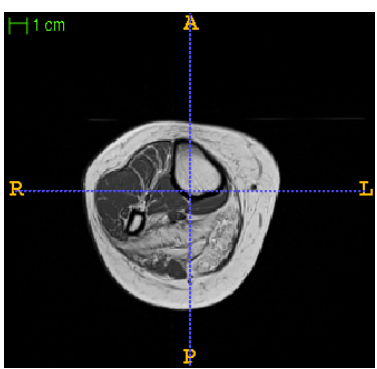

(b) DM1 patient

Fig. 1. T1-weighted MR images of the calf from the two neuromuscular diseases. On the left, Fig. 1(a), a slice of the MR image from a patient with facioscapulohumeral muscular dystrophy (FSH) and on the right, Fig. 1(b), a slice of the MR image from a patient with myotonic muscular dystrophy type 1 (DM1). These diseases are not readily distinguishable by eye.

i) MR Imaging with particular emphasis on Diffusion Tensor Imaging, which has been successfully used in neuroimaging, and ii) a novel structured sparsity machine learning algorithm, the $k$-support regularized SVM.

Diffusion Tensor Imaging is an imaging modality that captures the diffusion of water in tissues, and along with it, important structural information. It has been widely used in the study of the connectivity of the human brain [1]. Nonetheless, it has also been used in different clinical scenarios. Among them, one may cite the study of the human tongue [2], the heart muscle [2] and the human calf muscle [3]. DTI can capture important structural information in the case of the muscle. This is due to the fact that muscles are highly organized structures that present an architecture of elongated myofibers. Because myopathies affect the muscles, one may expect that the diffusion properties in diseased subjects are also altered [4].

Pattern classification techniques are widely applied in medical image processing. Along with DT imaging, pattern classification has gained widespread acceptance in neuroimaging studies because of its ability to capture multivariate relationships that characterize group differences. Some important examples of their application in neuroimaging scenarios include the study of Alzheimer's disease [5], male-female or older-younger classification [6], temporal classification of block design fMRI data [7] or the study of autism spectrum disorder [8].

In this work, we are interested in verifying that different myopathies alter muscle in distinct ways. Moreover, we investigate the discriminative power of diffusion and structural MR features in distinguishing between diseases. Fig. 1 shows the T1-weighted MR images of the two myopathies under investigation. The two images are very similar, making the distinction between them a very challenging task. In order to achieve this goal, we develop a strategy that exploits the rich information that is captured by both structural data (T1- and T2-weighted MR images) and DTI data to fuel state-of-the-art machine learn- 

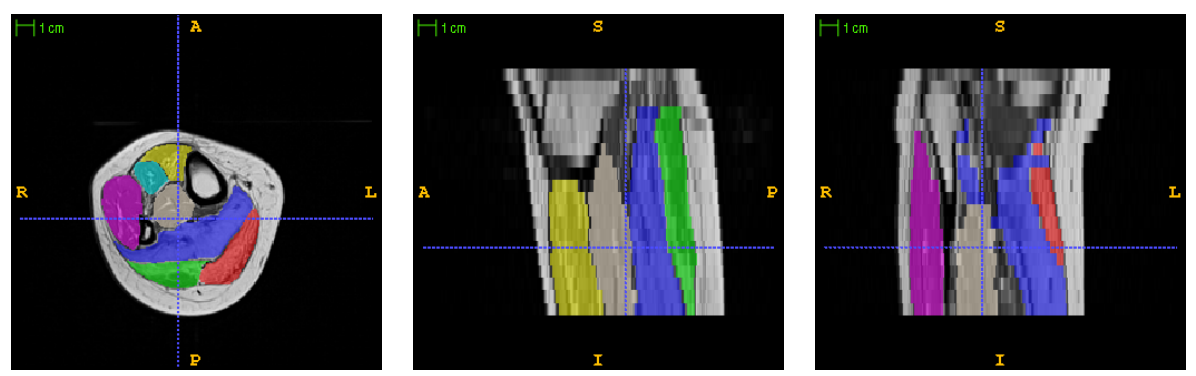

Fig. 2. An example of an T1 weighted MR image with the seven segmented muscles of the calf. Each color represents a single muscle. Yellow represents the anterior tibialis, cyan the extensor digitorum longus, magenta the peroneous longus, white the posterior tibialis, blue the soleus, green the lateral gastrocnemius, and red the edial gastrocnemius. (Figure best viewed in color.)

ing techniques. The use of high dimensional pattern classification in conjuction with DTI information has been previously investigated $[9,10,8]$. Nonetheless, it has been mainly applied to distinguish patients from controls in neuroimaging studies. Discriminating between patients poses additional challenges.

\section{Materials and Methods}

\subsection{Data description}

In this study, we have used a dataset consisting of twenty five subjects, 10 subjects were affected by FSH and 15 subjects were affected by DM1. In a clinical context, this is a large sample size. The subjects were imaged in the calf using a $1.5 \mathrm{~T}$ MRI scanner. Diffusion weighted images were acquired using the following parameters: repetition time $(T R)=3600 \mathrm{~ms}$, echo time $(T E)=70 \mathrm{~ms}$, slice thickness of $7 \mathrm{~mm}$ and $\mathrm{b}$ value of $700 \mathrm{~s} / \mathrm{mm}^{2}$ with 12 gradient directions and 13 repetitions. Diffusion tensors were estimated with the use of medInria software. ${ }^{5}$ The obtained volumes had a size of $64 \times 64 \times 20$ voxels and a voxel resolution of $3.125 \mathrm{~mm} \times 3.125 \mathrm{~mm} \times 7 \mathrm{~mm}$. T1- and T2-weighted MR images were acquired at the same time. As a consequence, the image volumes are naturally co-registered.

\subsection{Structural and diffusion features}

The images were segmented by an expert in the following 7 classes/muscle groups: 1) soleus (SOL), 2) lateral gastrocnemius (LG), 3) medial gastrocnemius (MG), 4) posterior tibialis (TP), 5) anterior tibialis (AT), 6) extensor digitorum

\footnotetext{
${ }^{5}$ http://med.inria.fr/
} 
longus (EDL), and 7) peroneous longus (PL). An example of the segmented muscle can be seen in Fig. 2. It is planned to automate this process in future work. In the meantime, the approach provides a strategy to avoid an invasive biopsy.

For every anatomical region, we extracted features from both the structural and the diffusion data. From the structural data, we extracted for every muscle: 1) the absolute volume, 2) the mean T1 signal, 3) the mean T2 signal, and 4) the Signal to Noise Ration (SNR). For the diffusion, we calculated for every muscle the mean values of the following scalar measures: 1) the Fractional Anisotropy (FA), 2) the trace of the diffusion tensor, 3) the volume of the tensor, 4) the eigenvalues (L1, L2, L3), 5) the planar coefficient (Cp), and 6) the linear coefficient $(\mathrm{Cl})$. These scalar measures were estimated with the use of medInria software. The resulted 84 variables were whitened and centered prior to applying the learning algorithms.

\section{$2.3 \quad k$-support regularized SVM}

Our proposed method, the $k$-support regularized SVM, is a novel algorithm based on the recently introduced $k$-support norm by Argyriou et. al [11]. The $k$-support norm is a sparsity regularization method that balances the $\ell_{1}$ and $\ell_{2}$ norms over a linear function in order to prevent over-fitting, similar to the well known elastic net [12]. The $k$-support norm can be computed as

$$
\|w\|_{k}^{s p}=\left(\sum_{i=1}^{k-r-1}\left(|w|_{i}^{\downarrow}\right)^{2}+\frac{1}{r+1}\left(\sum_{i=k-r}^{d}|w|_{i}^{\downarrow}\right)^{2}\right)^{\frac{1}{2}}
$$

where $|w|_{i}^{\downarrow}$ is the $i$ th largest element of the vector and $r$ is the unique integer in $\{0, \ldots, k-1\}$ satisfying

$$
|w|_{k-r-1}^{\downarrow}>\frac{1}{r+1} \sum_{i=k-r}^{d}|w|_{i}^{\downarrow} \geq|w|_{k-r}^{\downarrow} .
$$

In contrast to elastic net, $k$-support norm uses a $\ell_{1}$ penalty only for the smallest components and $\ell_{2}$ penalty for the largest components. We define the $k$-support norm regularized SVM ( $k$ sup-SVM) as the following optimization problem:

$$
\begin{aligned}
\min _{w \in \mathbb{R}^{d}, b \in \mathbb{R}, \xi \in \mathbb{R}^{n}} & \lambda\|w\|_{k}^{s p}+\sum_{i=1}^{n} \xi_{i} \\
\text { s.t. } & y_{i}\left(\left\langle w, x_{i}\right\rangle+b\right) \geq 1-\xi_{i}, \quad \xi_{i} \geq 0, \quad \forall i .
\end{aligned}
$$

This learning algorithm uses the hinge loss as in a classical SVM [13], but employs the $k$-support norm as a structured sparsity regularizer. This enables the learning algorithm to select a sparse but correlated subset of discriminative variables. $k$ sup-SVM has two input parameters, the $\lambda>0$ regularization parameter and $k \in\{1, \ldots, d\}$, where $d$ is the dimension of the feature space, the parameter that negatively correlates with the sparsity. 


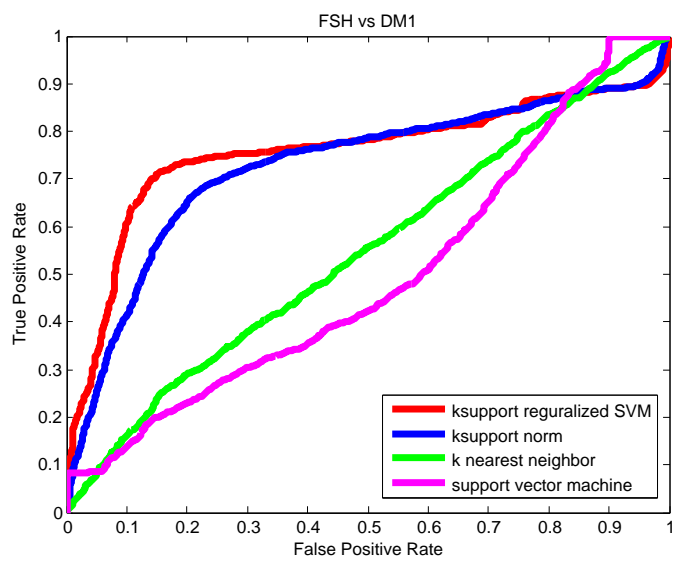

Fig. 3. Mean ROC curves for each classifier over 1000 trials. $k$ sup-SVM, shown in red, outperforms the rest of the methods $k$-support norm regularized squared error (blue), $k$ nn (green) and SVM (magenta). To the best of our knowledge, these are the first results presenting a significant discrimination between FSH and DM1 using MRI based markers. (Figure best viewed in color.)

Table 1. Classification mean accuracy (in $\% \pm$ standard error) and the mean area under the curve of all methods over 1000 trials. Chance is $60 \%$.

\begin{tabular}{|c||c|c|}
\hline Method & Accuracy & AUC \\
\hline \hline$k$ sup-SVM & $77 \pm 0.013$ & 0.756 \\
\hline$k$-support norm & $74 \pm 0.006$ & 0.726 \\
\hline$k$ nn & $61 \pm 0.015$ & 0.537 \\
\hline SVM & $59 \pm 0.015$ & 0.494 \\
\hline
\end{tabular}

\section{Results}

In order to explore the power of our proposed method we also train a number of supervised learning methods using the same features. More specifically, we examine the $k$ nearest neighbor algorithm [14], the support vector machine (SVM) [13] for a number of different kernels, and the $k$-support norm with squared loss as introduced by [11]. We examine the $k \mathrm{nn}$ algorithm with Euclidean distance and $k \in\{1,3,5,7,10\}$. For the SVM, we examine the following kernel functions, i) linear, ii) polynomial of third degree, and iii) radial basis function (RBF) with a soft-margin parameter $C \in\left\{10^{-3}, 10^{0}, 10^{3}\right\}$. For the $k$-support regularized squared loss and for the $k$ sup-SVM we examine the following combinations of parameters $\lambda \in\{1,10,1000\}$ and $k \in\{1,10,20,40,80\}$.

To approximate the generalization accuracy of the classification methods using the structural and DTI tensor features, we use a random splitting scheme with 1000 trials. In each trial, a random selection of $80 \%$ of the data are used to train the methods, while the remaining $20 \%$ are used to evaluate their performance. Model selection was performed in a similar fashion using only $80 \%$ of the data. We report only the generalization performance for each method.

Fig. 3 shows the mean ROC curve across all trials, while Table 3 gives the mean classification accuracy and area under the curve over 1000 trials. $k$ sup- 
SVM outperforms the rest of the methods by achieving a mean area under the curve (AUC) of 0.7141 and mean accuracy $77 \% \pm 0.013$. The $k$-support norm also performs well with a mean AUC of 0.694 and mean accuracy $72 \% \pm 0.006$, while $k \mathrm{nn}$ and SVM performances are near chance, which is $60 \%$. Moreover, with a Wilcoxon signed rank test we show that the $k$ sup-SVM is statistically significantly better than all other methods (all $p$-values were $\ll 10^{-9}$ ). Fig. 4 shows the boxplots of the weights of the structural and DTI tensor features selected by the $k$ sup-SVM over 1000 bootstrap trials. On each box, the central mark is the median, the edges of the box are the 25 th and 75 th percentiles, while the whiskers extend to the most extreme data points that are not considered outliers. Since the MRI features are evaluated for each of the seven muscles of interest, we plot them per muscle. A number of features are systematically assigned zero weight across multiple trials (green line), indicating that they do not provide useful information for the distinction of the two diseases, while the ones with non-zero weight are considered more informative.

Toward demonstrating the added value of the DTI features, we compared the performance of the $k$ sup-SVM when trained only on structural data against its previous result. The same experimental setting as before was used. In this case, $k$ sup-SVM achieved a mean AUC of 0.697 and mean accuracy $73 \% \pm 0.006$. According to a Wilcoxon signed rank test, this performance is statistically significantly worse than its previous performance using both structured and DTI features ( $p$-value was $\ll 0.05)$.

\section{Discussion}

An analysis of variables selected by the sparsity regularizer (Fig. 4) gives an indication that discrimination varies across muscles as well as features. Increased muscle volume in AT, EDL, MG, and TP was associated with DM1, while increased volume in PL and SOL was associated with FSH. T1 and T2 signal was consistently positively associated with FSH in the EDL muscle. A broad range of statistics were discriminative for the MG muscle, while discriminative features for most other muscles were comparatively sparse.

While MRI markers, and DTI tensor features in particular, have previously been shown to differ between disease and control subjects [4, Table 2], we are unaware of previous studies that have shown significant ability to discriminate between disease conditions. Indeed, a high-dimensional analysis of MRI based markers was required to achieve non-random performance in this more challenging task. Sparsity regularization appears to be a more important property of the learning algorithm than non-linearity, as evidenced by the comparatively stronger performance of $k$-support norm regularized SVM or squared error, as compared to a SVM with non-linear kernels or $k$ nearest neighbors (both nonsparse, non-linear algorithms).

In this paper, we have presented several novel methodological and clinical developments related to the use of pattern recognition methods in neuromuscular disease classification. While previous studies have focused on the comparatively 

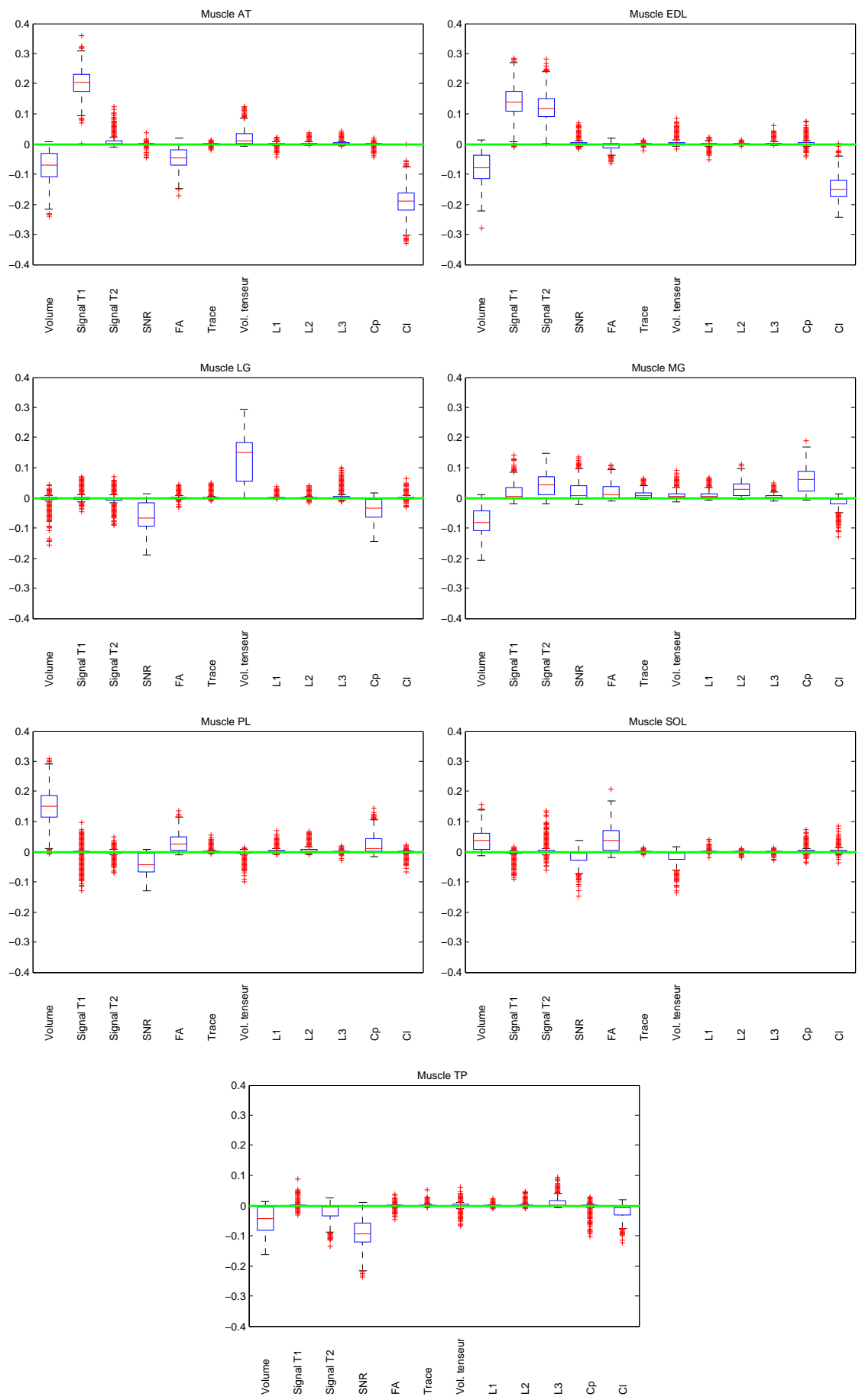

Fig. 4. Boxplot of the weights given to the structural and DTI features of the 7 muscles by the $k$ sup-SVM over 1000 trials. Positive values indicate positive association with FSH, while negative values indicate positive association with DM1. Values close to zero are indicative of a lack of discriminative information between the two disease conditions. 
easy task of separating disease from healthy subjects, we have approached the more difficult and clinically relevant task of discriminating between diseases. We have shown that a combination of T1- and T2-weighted MR images and Diffusion Tensor Imaging data are discriminative for separating patients with facioscapulohumeral muscular dystrophy and Myotonic muscular dystrophy type 1. Our novel machine learning algorithm, the $k$ sup-SVM, is an essential machine learning approach for achieving the best performance, with a mean accuracy of $77 \% \pm 0.013$.

\section{References}

1. Le Bihan, D., Mangin, J.F., Poupon, C., Clark, C.A., Pappata, S., Molko, N., Chabriat, H.: Diffusion tensor imaging: concepts and applications. Journal of magnetic resonance imaging 13 (2001) 534-546

2. Gilbert, R.J., Napadow, V.J.: Three-dimensional muscular architecture of the human tongue determined in vivo with diffusion tensor magnetic resonance imaging. Dysphagia 20 (2005) 1-7

3. Galban, C.J., Maderwald, S., Uffmann, K., de Greiff, A., Ladd, M.E.: Diffusive sensitivity to muscle architecture: a magnetic resonance diffusion tensor imaging study of the human calf. European journal of applied physiology 93 (2004) 253-262

4. Qi, J., Olsen, N.J., Price, R.R., Winston, J.A., Park, J.H.: Diffusion-weighted imaging of inflammatory myopathies: Polymyositis and dermatomyositis. Journal of Magnetic Resonance Imaging 27 (2008) 212-217

5. Klöppel, S., Stonnington, C.M., Chu, C., Draganski, B., Scahill, R.I., Rohrer, J.D., Fox, N.C., Jack, C.R., Ashburner, J., Frackowiak, R.S.: Automatic classification of $\mathrm{mr}$ scans in alzheimer's disease. Brain 131 (2008) 681-689

6. Lao, Z., Shen, D., Xue, Z., Karacali, B., Resnick, S.M., Davatzikos, C.: Morphological classification of brains via high-dimensional shape transformations and machine learning methods. Neuroimage 21 (2004) 46-57

7. LaConte, S., Strother, S., Cherkassky, V., et al.: Support vector machines for temporal classification of block design fmri data. NeuroImage 26 (2005) 317

8. Ingalhalikar, M., Parker, D., Bloy, L., Roberts, T.P., Verma, R.: Diffusion based abnormality markers of pathology: Toward learned diagnostic prediction of asd. Neuroimage 57 (2011) 918-927

9. Caan, M., Vermeer, K., Van Vliet, L., Majoie, C., Peters, B., den Heeten, G., Vos, F.: Shaving diffusion tensor images in discriminant analysis: A study into schizophrenia. Medical Image Analysis 10 (2006) 841-849

10. Wang, P., Verma, R.: On classifying disease-induced patterns in the brain using diffusion tensor images. MICCAI (2008) 908-916

11. Argyriou, A., Foygel, R., Srebro, N.: Sparse Prediction with the k-Support Norm. In: Advances in Neural Information Processing Systems (NIPS). (2012)

12. Zou, H., Hastie, T.: Regularization and variable selection via the Elastic Net. Journal of the Royal Statistical Society, Series B 67 (2005) 301-320

13. Cortes, C., Vapnik, V.: Support-vector networks. Mach. Learn. 20 (1995) 273-297

14. Wang, P., Gur, R., Verma, R.: A novel framework for identifying dti-based brain patterns of schizophrenia. ISMRM, Toronto (2008) 3-9 